\title{
Germanica
}

\section{La confrontation littéraire au passé national- socialiste dans le contexte de la RDA des années}

\section{0}

L'exemple de Klaus Schlesinger

Die erzählerische Auseinandersetzung mit der NS-Vergangenheit im Kontext der DDR der 1960er Jahre : der Fall Klaus Schlesinger

\section{Daniel Argelès}

\section{OpenEdition}

\section{Journals}

Édition électronique

URL : http://journals.openedition.org/germanica/520

DOI : 10.4000/germanica.520

ISSN : 2107-0784

Éditeur

Université de Lille

\section{Édition imprimée}

Date de publication : 1 juin 2008

Pagination : 119-141

ISBN : 978-2-913857-21-6

ISSN : 0984-2632

\section{Référence électronique}

Daniel Argelès, «La confrontation littéraire au passé national-socialiste dans le contexte de la RDA des années 1960 », Germanica [En ligne], 42 | 2008, mis en ligne le 01 juin 2010, consulté le 06 octobre

2020. URL : http://journals.openedition.org/germanica/520 ; DOI : https://doi.org/10.4000/germanica. 520

Ce document a été généré automatiquement le 6 octobre 2020.

(c) Tous droits réservés 


\title{
La confrontation littéraire au passé national-socialiste dans le contexte de la RDA des années 1960
}

\author{
L'exemple de Klaus Schlesinger
}

Die erzählerische Auseinandersetzung mit der NS-Vergangenheit im Kontext der DDR der 1960er Jahre : der Fall Klaus Schlesinger

\section{Daniel Argelès}

1 Entre 1960 et 1971, l'écrivain Klaus Schlesinger (1937-2001) publie trois textes sur le " passé » national-socialiste. Il y est question du génocide perpétré par le $\mathrm{III}^{\mathrm{e}}$ Reich contre les Juifs d'Europe, de la question de la culpabilité individuelle et collective des "pères » et de son héritage par la deuxième génération, à savoir celle des Allemands trop jeunes pour s'être rendus directement coupables de ces crimes.

Ce travail de retour sur le passé intervient très tôt dans son œuvre, puisqu'il s'agit des trois premiers textes de fiction qu'il publie. La nouvelle «David», qui raconte l'interrogatoire d'un enfant du ghetto de Varsovie par un soldat allemand, paraît en 1960 dans Neue Deutsche Literatur (NDL), la revue de l'Union des Écrivains de RDA ${ }^{1}$. Cinq ans plus tard, NDL publie sa deuxième nouvelle, intitulée "Michael ", où un jeune Allemand de l'Est croit reconnaître son père sur une photo d'exécution de villageois juifs en Pologne et s'interroge sur son implication dans les crimes nazis ${ }^{2}$. En 1971, il publie son troisième texte de fiction, le roman Michael, qui développe et parachève l'esquisse précédente ${ }^{3}$.

Cette confrontation avec le passé a une dimension personnelle forte : si Schlesinger n'a lui-même que huit ans à la fin de la guerre, son père, qui disparaît lors des derniers jours de la bataille de Berlin, est membre du NSDAP. Dans un entretien de 2001, il se souvient d'avoir été un «jeune nazi »: «À sept ans, je croyais en Hitler, et je croyais que l'Allemagne ne pourrait jamais perdre la guerre $»^{4}$. En avril 1945, dans « la ville encerclée et prise sous le feu de l'artillerie », il entrait encore chez le marchand de légumes de sa rue «en claquant les talons, en dressant le bras droit en l'air et en 
s'écriant 'Heil Hitler!' »'. Aussi ces trois récits ont-ils une dimension cathartique : dans l'entretien de 2001, il confie s'être libéré « l'âme » de sa « névrose » par l'écriture ${ }^{6}$.

4 Ces textes, pourtant, occupent une place injustement méconnue dans l'histoire de la littérature allemande d'après-guerre et de la confrontation littéraire au nationalsocialisme. Certes, Emmerich mentionne bien l'auteur de Michael aux côtés de Rolf Schneider, de Jurek Becker ou de Christa Wolf comme exemple du fait que «la jeune génération de la RDA était également concernée par le problème du nazisme », mais il se contente d'en résumer l'argument en quelques lignes ${ }^{7}$. Dans son ouvrage sur l'image des Juifs dans la littérature de RDA, O'Doherty ne mentionne pas «David» alors que plusieurs auteurs, dont Willy Bredel ou Franz Fühmann, sont abordés pour une simple nouvelle ${ }^{8}$. Dans son livre sur la Väterliteratur des années 1970 et 1980, Mauelshagen est une des rares à aborder Michael sur plus d'une page et à en souligner le caractère "précoce ", «très réussi» voire "virtuose» et, surtout, "singulier» dans sa démarche9. Quant à Köhler, si elle fait bien ressortir la place essentielle de l'histoire dans son œuvre, elle laisse malheureusement «David» de côté et, soucieuse de souligner les liens des deux versions de « Michael » avec le reste de l'œuvre, elle passe rapidement sur le contexte des années 1960 dans lequel elles sont écrites ${ }^{10}$.

$5 \quad$ L'objectif de cet article est double : d'abord, rendre justice à ces textes en soulignant en effet leur singularité, leur valeur littéraire et l'intégrité de leur démarche de confrontation au passé. Ensuite, observer l'évolution du jeune écrivain et de son travail dans le contexte de la RDA. Car s'il parvient à s'en émanciper et aboutit à une approche originale et non stéréotypée, il est clair que ses textes naissent et sont publiés dans un environnement qui les marque: celui de l'antifascisme promu par le régime estallemand. Nous tenterons en particulier de repérer les stratégies d'écriture par lesquelles il se libère de ces déterminations. Car en s'efforçant de mener à bien une confrontation au passé qui échappe au dogmatisme, à l'instrumentalisation politique et aux conclusions prédéterminées, Schlesinger s'engageait déjà dans une logique d'émancipation esthétique, éthique et politique.

\section{I. « David »(1960)}

6 Dans l'entretien de 2001, Schlesinger répond à la question «Qu'est-ce qui vous a amené à écrire? » en soulignant son rapport au national-socialisme et à la Shoah : « Assez tôt, en tant que jeune garçon, j'ai eu entre les mains une brochure sur un camp de concentration, Sachsenhausen ou Buchenwald. C'est ainsi que je suis tombé sur cette problématique qui ne m'a dès lors plus quitté. J'étais un jeune nazi, si l'on veut. À sept ans, je croyais en Hitler, et je croyais que l'Allemagne ne pourrait jamais perdre la guerre. Pour moi, la fin de la guerre a été un effondrement, et pas seulement parce que j'ai perdu mon père, qui était encore vivant une semaine avant - il a longtemps été considéré comme disparu, et j'ai toujours espéré qu'il reviendrait. Donc, je me suis très fortement confronté à ce que l'on appelle fascisme ou national-socialisme, j'ai lu tout ce qui paraissait, je me suis procuré des livres de Pologne, du Centre culturel polonais, et aussi de l'Ouest [...]. Je lisais beaucoup, je me suis intéressé au ghetto de Varsovie et j'ai même essayé, à l'époque, d'apprendre le yiddish. J'étais capable de lire des journaux dans cette langue, je m'étais d'ailleurs abonné à un journal en yiddish de Varsovie. Puis j'ai écrit une histoire, peut-être inspirée de certaines histoires du ghetto. On y trouve 
un motif que j'ai souvent réutilisé plus tard, celui d'un enfant trahi par des adultes. La nouvelle s'appelle 'David' (...). »"11

7 L'écriture apparaît donc liée, chez le jeune Schlesinger, à l'« effondrement » de son univers d'enfant au moment de la défaite : à la perte du père, au choc de la découverte des crimes commis au nom d'un régime jusque-là admiré et au renversement complet des valeurs qui s'en est suivi. Les fondements existentiels de l'acte d'écrire sont donc complexes. La Shoah y joue un rôle déterminant parce qu'elle est au cœur de ce retournement: c'est au moment de la découverte des crimes que le régime adulé auquel son père avait fait allégeance devient le régime le plus inhumain et le père décédé un problème. De 1945 à 1960, ce "complexe» - cette "névrose ", pour reprendre ses termes - se nourrit de lectures sur le sujet, nombreuses dans l'environnement antifasciste de la zone soviétique et de la RDA où vivent l'enfant et le jeune homme, et auxquelles s'ajoutent des lectures polonaises et occidentales.

Puis vient, très vraisemblablement, le déclic. Si nous n'en avons pas trouvé confirmation dans les témoignages écrits laissés par l'auteur, les indices concordent néanmoins pour souligner le rôle essentiel joué par une photo dans le passage à l'écriture : celle du «plus jeune combattant du soulèvement du ghetto de Varsovie " à qui la nouvelle est dédiée, Szanan Lent $(99)^{12}$. La photo, en noir et blanc, figure dans un ouvrage édité par l'Institut historique juif de Varsovie et paru à Berlin en 1960 sous le titre Faschismus, Getto, Massenmord. Dokumentation über Ausrottung und Widerstand der Juden in Polen während des Zweiten Weltkrieges ${ }^{13}$. Elle y côtoie celle de son père, Hersz Lent, membre d'un parti sioniste de gauche, et celles de plus de cinquante résistants juifs de Pologne. La légende indique: "Szanan Lent, fils de Hersz, le plus jeune des insurgés (14 ans), membre de l'organisation communiste de combat de la jeunesse, tombé pendant le soulèvement $»^{14}$. Si nous parlons de déclic - au-delà de l'évident effet de miroir provoqué par l'axe père-fils, sur lequel nous reviendrons -, c'est que cet ouvrage est le seul document en relation avec la nouvelle conservé au fonds d'archives Klaus Schlesinger de l'Académie des Arts à Berlin. Il n'est pas anodin que, quarante ans plus tard, au terme d'une vie mouvementée entre Est et Ouest où beaucoup de documents se sont perdus, Schlesinger l'ait encore eu en sa possession.

\section{Le contexte « antifasciste »}

9 Avant d'en venir aux enjeux existentiels, littéraires et idéologiques de la nouvelle, il convient de s'arrêter un instant sur ce livre, qui en dit long sur le contexte dans lequel Schlesinger écrit. Travail sérieux de documentation sur la Shoah, il contient près de 500 documents d'archives (dont 231 images et fac-similés) provenant de l'Institut historique juif de Varsovie ou de la Commission centrale d'investigation des crimes nazis en Pologne, de divers échelons de l'administration national-socialiste, des procès de Nuremberg ou de Pologne, etc. En cela, il réactive la tradition des ouvrages de vulgarisation publiés après-guerre en RDA par l'Association des persécutés du régime nazi (Vereinigung der Verfolgten des Naziregimes, VVN) jusqu'à sa dissolution en 1953 et rappelle un versant sincère de l'engagement antifasciste dans lequel nombre de rémigrants juifs ou non-juifs avaient pu se reconnaitre et qui, jusqu'au tournant de 1953, avait pu faire apparaître l'État est-allemand comme un antidote à l'antisémitisme et au national-socialisme ${ }^{15}$. 

l'instrumentalisation de l'antifascisme par le régime est-allemand. L'introduction signée du directeur de publication, relativement factuelle, reste encore mesurée à cet égard. D'orientation " progressiste », elle retrace l'historique de la " solution finale » et de la résistance des Juifs qui "combattirent en Pologne sur tous les fronts de la résistance antifasciste » (22), perpétuant « les traditions progressistes des Juifs polonais luttant pour la libération nationale et sociale » (25). L'instrumentalisation s'y limite à un paragraphe, codicille militant où toutes les menaces de résurgence deviennent l'apanage de la RFA: «En RFA, les partisans du néofascisme redressent de plus en plus la tête. Les vieux généraux d'Hitler reconstruisent une Wehrmacht maintenant dotée, de surcroît, de la terrible arme nucléaire. Simultanément, nous assistons aux efforts de ces mêmes forces réactionnaires pour dissimuler la vérité sur le génocide perpétré par le Troisième Reich en répandant l'opinion selon laquelle l'extermination de 6 millions de Juifs n'existerait que dans la propagande et ne serait qu'une calomnie » (25-26).

11 Cette introduction, cependant, est précédée d'un avant-propos beaucoup plus univoque signé par «la maison d'édition » où la RDA est décrite comme l'État qui «préserve l'honneur et la dignité de la nation allemande» (11). «En République Démocratique Allemande, l'impérialisme a été éliminé. Ainsi, fascisme, racisme et antisémitisme ont été privés de leur terreau. En RDA, il n'y a pas de cimetières juifs et de synagogues profanés, pas de murs couverts de croix gammées et de menaces contre les concitoyens juifs. Il n'y a pas d'enseignants qui se vantent d'avoir perpétré des atrocités contre les Juifs » (11). L'instrumentalisation à des fins de politique extérieure s'accompagne donc d'un dédouanement de la RDA et de ses citoyens, exonérés de l'héritage du passé16. S'y ajoute une hiérarchisation des victimes où les antifascistes prennent le pas sur les "persécutés raciaux». Dans la guerre du fascisme contre «l'ennemi intérieur », la hiérarchie est claire : «La persécution des communistes a occupé la première place ; les actions contre la population juive sont venues à côté (nebenher)» (10). Emphatique dans l'invocation de la Shoah - «les barbares hitlériens ont assassiné 6 millions de Juifs»-, le texte marginalise en même temps les Juifs: les communistes "ne se contentèrent pas de prévenir, ils organisèrent la résistance contre le fascisme, et ce sont eux qui firent les plus grands sacrifices (brachten die meisten Opfer) » (11) ${ }^{17}$.

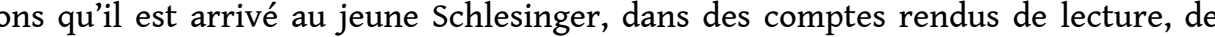
dénoncer lui aussi certaines continuités biographiques entre le III ${ }^{e}$ Reich et la RFA : dans le Beuth-Spiegel, par exemple, la revue d'étudiants de l'école d'ingénieur de Berlin-Ouest où il suivait des cours du soir ${ }^{18}$, ou bien dans la revue médicale humanitas, où il rappelle la carrière de maire et de député au parlement de Kiel du «boucher de Varsovie ", l'ancien général SS Heinz Reinefarth ${ }^{19}$. À cette occasion, un certain langage antifasciste se retrouve sous sa plume, par exemple lorsqu'il attribue au livre qu'il recense le désir «d'alerter face à l'impérialisme allemand, racine de tout le mal fasciste ». Mais cette critique de la RFA des années 1950 ne se transforme pas en exonération de la RDA ou en hiérarchisation des souffrances et des résistances.

\section{Le dépassement de la vulgate antifasciste dans la nouvelle}

Situé dans le ghetto de Varsovie, le texte raconte les derniers instants d'un enfant juif de neuf ans dont les parents et le frère ont été déportés et qui, lors du soulèvement, est chargé par son oncle et un commandant de la résistance de transmettre un message 
chiffré à d'autres combattants du ghetto. Pris par les Allemands, David subit l'interrogatoire violent d'un sous-officier et d'un soldat, jusqu'à ce qu'un lieutenant, gêné dans ses méditations par les cris de l'enfant et les hurlements de son interrogateur, se décide à intervenir lui-même. Pour endormir la méfiance de David, qui revient peu à peu à lui, l'officier lui parle avec douceur, puis lui montre une photo de famille où l'on voit son petit dernier («du même âge que toi », lui dit-il) avec un ballon de football sous le bras (109).

Affaibli par l'interrogatoire, David succombe un instant aux manœuvres de l'officier : il est pris successivement d'un désir de père - «Cet officier pourrait être ton père, pensat-il, et il souhaita un instant qu'il en fût ainsi » - , d'un désir de famille - « Tu aurais de nouveau des frères, tu pourrais jouer au ballon avec eux »-, d'un désir d'adoption - « Il s'imagina en train d'habiter avec toute sa famille dans une maison n'appartenant qu'à eux. Il y aurait là l'oncle Dolek, la mère, l'officier, et il aurait une chambre pour lui tout seul »-, et d'un désir de défection - «Il fait chaud, nous enlevons nos vestes, les posons sur la chaussée, elles forment les buts à cinq pas l'une de l'autre... L'oncle Dolek sort du vestibule, il porte un uniforme allemand. Il me prend par la main... » (110-111)

Mais, au moment où David va céder, une attaque aérienne soviétique vient enrayer la stratégie de l'officier. L'enfant reconnait la supercherie, rassemble « toutes ses forces et tout son courage, se lève et crache au visage de l'officier ", signant ainsi son arrêt de mort. Voyant venir à lui le premier interrogateur, David pense "à la jubilation avec laquelle Oncle Dolek et les camarades avaient accueilli la première attaque aérienne soviétique, et l'explosion proche des bombes lui apparut comme le fanal de la rédemption » (112).

16 La première nouvelle de Schlesinger porte donc la marque du contexte où elle a été écrite et publiée. Le ghetto de Varsovie, son soulèvement, la résistance font en effet partie des figures privilégiées des récits antifascistes publiés en RDA, qu'il s'agisse de biographies ou, à l'instar de l'ouvrage mentionné plus haut, de recueils de témoignages ou de documents. Certes, l'identité communiste de Szanan Lent, le modèle de David, n'est pas mentionnée. De même, l'appartenance politique de l'oncle, du " commandant " ou même des "camarades" auprès desquels David a trouvé refuge n'est pas explicitée, ces termes pouvant renvoyer à plusieurs groupes ou sous-groupes armés du ghetto. Mais rien n'interdit de situer l'acte de résistance de David dans la mouvance communiste du soulèvement. En outre, la nouvelle se termine par un bombardement soviétique de Varsovie qui tend à inscrire son sacrifice dans le sens de l'histoire véhiculé par le régime et la littérature antifasciste.

17 Il est à noter, cependant, que Schlesinger n'invente pas ce bombardement, mais qu'il s'inspire vraisemblablement de l'histoire du soulèvement du ghetto de Varsovie écrite par Bernard Mark, historien polonais membre de l'Institut historique juif de Varsovie qui publiera également Faschismus, Getto, Massenmord ${ }^{20}$. Dans cet ouvrage, clairement situé dans la lignée de l'antifascisme, Mark s'appuie sur des témoignages de survivants (dont celui du général SS Stroop) pour décrire l'attaque aérienne soviétique du 13 mai 1943. Une photo de Szanan Lent y figure également, ainsi que quelques mots sur sa participation aux combats, sa mort et sa décoration posthume. Une phrase du livre "Les combattants du ghetto saluèrent l'attaque aérienne soviétique avec une grande joie et satisfaction" (335) - a même manifestement trouvé un écho à la fin de la nouvelle. 
18 Le texte de Schlesinger a donc été nourri et inspiré par toute une littérature antifasciste. L'hommage à la résistance et la fin du texte ne pouvaient déplaire aux autorités littéraires de la RDA. Et c'est sans doute sous ce jour que le comité de lecture de NDL, la revue de l'Union des Écrivains, l'a lu et publié : comme une nouvelle d'inspiration antifasciste écrite par une jeune plume prometteuse. Plume qui, pour ne rien gâter, travaillait dans la «production ». À défaut d'être ouvrier, Schlesinger avait l'avantage d'être laborantin : le promouvoir correspondait aux exigences formulées un an avant lors de la conférence de Bitterfeld d'avril 1959.

19 «David », cependant, ne se résume pas, loin s'en faut, à une illustration littéraire de la vulgate antifasciste. D'abord, parce que le texte n'est à aucun moment suspect de hiérarchisation ou de marginalisation des victimes. Le cadre historique est clairement celui de la Shoah: la déportation et la liquidation du ghetto de Varsovie y sont évoquées sans ambiguïté. David est victime en tant que Juif, et il reste au centre de la nouvelle. Il se distingue en outre très nettement de l'autre figure d'enfant qui devait s'imposer à peu près au même moment comme icône de la littérature antifasciste : l'enfant passif, muet, quasi animal sauvé par la résistance communiste du camp de Buchenwald dans le roman de Bruno Apitz: Nackt unter Wölfen ${ }^{21}$. À la différence de ce dernier, David est acteur de son destin. Assassiné dans le cadre du génocide, il se bat et choisit délibérément de mourir pour la résistance. À cet égard, le texte de Schlesinger est important : même si la lutte des résistants juifs communistes faisait partie des topoi de l'antifascisme, une telle figure se retrouve rarement dans la littérature allemande d'après-guerre, a fortiori chez un auteur non juif 22 .

20 Enfin, et c'est là l'essentiel, la nouvelle dépasse l'antifascisme primaire par la force et la complexité émotionnelles de sa construction. À l'hommage à un enfant juif, victime de la Shoah et héros du combat antifasciste, s'ajoute une pluridimensionnalité étonnante $\mathrm{du}$ dispositif narratif. La clef se résume à une phrase : «Cet officier pourrait être ton père ». Cette pensée de David dit d'abord tout le sentiment d'abandon, toute la détresse, la fragilité de l'enfant livré à ses bourreaux. Elle dit aussi, en creux, l'horreur de ces crimes contre l'humanité commis par des êtres "normaux", pères aimants dans le civil $^{23}$ : ici, l'officier pervertit l'amour paternel en faisant de la photo de son fils une arme de trahison. Rapportée aux années 1960, elle dit enfin toute la question de la culpabilité : la phrase peut être comprise du point de vue de l'auteur, comme un mea culpa (mon père a sans doute été un de ces officiers), ou du point de vue du lecteur, comme une invitation faite à la deuxième génération à s'interroger sur le rôle exact joué par ses parents pendant le III ${ }^{\mathrm{e}}$ Reich.

21 Les lignes potentielles d'identification sont donc multiples et donnent à la nouvelle une force étonnante. Le lecteur se retrouve en présence de deux doubles de l'auteur : deux enfants de l'âge qu'avait Schlesinger à l'époque. Le premier, David, est adopté par la logique émotionnelle du texte, logique confirmée par le prénom que Klaus Schlesinger donne deux ans plus tard à son premier fils: David. Un acte fort (d'ailleurs pas nécessairement facile à porter pour le fils) dont on devine les motivations : demander pardon et offrir réparation par l'adoption symbolique d'une victime représentative de l'ensemble des victimes; l'intégrer à la filiation (familiale et nationale) là où le père l'excluait jusqu'à l'élimination; rompre le fil de la culpabilité en soustrayant symboliquement son fils au continuum des générations.

22 La question de l'héritage du génocide est au cœur du texte, incarnée par la présence sur les lieux du deuxième double de l'auteur : en montrant la photo de son fils à David, en 
instrumentalisant son image à des fins criminelles, l'officier l'implique dans l'histoire. La photo incarne la nature et le degré de complicité, ou plutôt de co-culpabilité, de la deuxième génération. En écrivant cette nouvelle après avoir scruté les visages de Szanan et de son père dans les ouvrages d'histoire de l'époque et en plaçant entre les mains d'un père criminel une photo de soi enfant, cliché de bonheur familial proclamant une innocence éternelle, Schlesinger rappelle que le devoir de mémoire a deux versants, représentés ici par les deux enfants : la confrontation aux photos des victimes - donc à l'horreur des crimes commis - et la confrontation aux photos de famille - donc à l'horreur des bonheurs du quotidien vécus en parallèle.

On voit donc que Schlesinger se tient à l'écart de toute forme d'exonération des Allemands de l'Est et de leur pays. Antifasciste à certains égards, son récit se démarque clairement de l'idéologie officielle qui tente de soustraire la RDA à l'héritage du passé : il pose au contraire, implicitement mais sans ambiguïté, la question du devenir de ces pères et de ces fils après la guerre. Ce n'est pas un hasard si, dans la foulée de " David ", Schlesinger écrit " Michael », nouvelle où un Allemand de l'Est se confronte à son père après avoir cru le reconnaître sur une photo d'exécution de villageois en Pologne ${ }^{24}$. Car ces deux textes, dont les titres nominaux renvoient au peuple juif et au peuple allemand, représentent les deux faces d'une même question. On ne peut rendre hommage à David sans impliquer le "Michel allemand», et on ne peut parler de Michael, fût-il est-allemand, sans penser à David.

\section{II. « Michael » (I'« ébauche » de 1965)}

Paru également dans la revue NDL, le deuxième texte de fiction de Schlesinger n'est pas un chef d'œuvre majeur de la littérature d'après-guerre, mais il reste passionnant par son sujet et les risques idéologiques et esthétiques qu'il représente.

"Michael », en effet, aborde un sujet tabou : la présence d'anciens criminels nazis en RDA. Le discours antifasciste officiel, nous l'avons dit, tendait à entretenir le mythe d'une innocence intrinsèque de l'État est-allemand et de ses citoyens et à réserver ces questions à la République Fédérale. Au moment de la publication de "Michael », en mai 1965, le premier procès de Francfort continue de secouer la RFA, et la RDA de cultiver son image d'État supposément exemplaire. Le régime réserve ainsi un accueil très favorable à Die Ermittlung et à son auteur Peter Weiss, qui participe en mai, à Berlin-Est, à une rencontre internationale d'écrivains à l'occasion du $20^{\mathrm{e}}$ anniversaire de la fin du régime national-socialiste ${ }^{25} .1965$ est également l'année où les éditions d'État de la RDA publient le fameux "Livre brun », ouvrage dénonçant les fonctions au sein du NSDAP ou de la SS de 1800 dirigeants économiques, hommes politiques et hauts fonctionnaires de la $\mathrm{RFA}^{26}$. Le dogme d'une supériorité de l'État antifasciste sur un voisin compromis n'est donc nullement remis en cause, et la question de survivances nazies en RDA reste largement taboue ${ }^{27}$.

Aussi la nouvelle est-elle très encadrée. Présentée dans une rubrique intitulée « Neue Namen ", elle est accompagnée d'une critique du texte signée du rédacteur en chef adjoint de la revue, Heinz Plavius, et d'un droit de réponse de l'auteur. Mais elle est publiée : l'objectif de cette "expérience " (182) est aussi de désamorcer à l'avance les critiques, de s'en prémunir en donnant un certain nombre de gages aux instances gardiennes de l'orthodoxie ${ }^{28}$. Dans son livre sur le modernisme et la politique culturelle de la RDA, Günter Erbe range Plavius dans la «nouvelle phalange de fonctionnaires de 
la culture » qui, à partir de 1963, « s'appliquent à concilier la nouvelle littérature avec les lignes directrices de Bitterfeld $»^{29}$.

\section{Une nouvelle sur un sujet tabou}

Écrite du point de vue du héros, mais à la $3^{e}$ personne, l'« ébauche » raconte deux, trois jours dans la vie de Michael Berger, jeune Allemand de l'Est de 23 ans aux « yeux d'un bleu magnifique », jeune ingénieur promis à de "grands espoirs» (166), qui, par un samedi ensoleillé, découvre au fond d'une librairie d'occasion un volume de documents sur l'occupation de la Pologne par les armées hitlériennes et les différents stades de l'extermination des Juifs d'Europe de l'Est. Parmi ces documents figure une photo d'exécution sommaire d'habitants juifs d'un village polonais sur laquelle il pense reconnaître son père au premier rang des soldats allemands.

Le récit aborde les principales étapes du parcours de Michael après cette découverte : une nuit d'insomnie, une journée d'errance en ville, entre café et métro aérien berlinois, une nuit passée dans l'appartement et les bras accueillants d'une serveuse, un dîner familial lourd de silence, puis la confrontation avec le père et la rupture : le départ du domicile parental. La narration, toutefois, n'est en rien linéaire. Elle consiste en une succession de fragments plus ou moins longs, stylistiquement hétérogènes et séparés par des titres. Des raisonnements abstraits sur la culpabilité alternent avec l'évocation de la scène d'exécution, des souvenirs d'enfance, un résumé du parcours du père, des monologues intérieurs hantés par les appels à la justice des victimes - les Jurek, David, Samuel scandant le nom de sa famille -, une restitution de la scène d'explication avec le père, le récit imaginaire d'autres confrontations, mi-avortées, mivirtuelles.

Le texte de Schlesinger fait donc preuve d'une certaine modernité littéraire, qui le distingue d'entrée des récits antifascistes plus traditionnels. Les références dont il se réclame - et qui lui donnent un côté programmatique - vont dans le même sens: Seghers et Hemingway y côtoient Prévert, des versets de la Bible et deux standards du jazz: Take the A-train et On the sunny side of the street. Dès la première page, le jeune auteur refuse les clivages littéraires de la guerre froide et l'obligation de choisir entre le réalisme antifasciste et la modernité littéraire occidentale : pour se confronter au passé, Michael devra ressortir le volume accusateur de l'étagère où il l'avait glissé, entre La septième croix et Le Soleil se lève aussi, donc "réunir ainsi de nouveau Hemingway et Anna Seghers » (156) ${ }^{30}$. Le texte a en outre un indéniable parfum de bohème où se mêlent bars de jazz, rencontres amoureuses sans lendemain, rébellion adolescente contre «l'autorité de l'âge, de l'expérience » (179). Enfin, la nouvelle est «moderne » par sa fin en suspens : au terme du récit, le lecteur sait seulement que Michael quitte le domicile parental; ses actions ultérieures restent en pointillés, invitation faite au lecteur à se mettre à sa place et à s'interroger sur soi-même et son propre rapport au passé.

Il s'agit donc clairement d'un texte qui flirte avec les limites officielles. Dès le deuxième chapitre, citant les programmes scolaires de RDA et le traitement du passé nationalsocialiste dans la presse du pays, Schlesinger écrit : «Comme pour presque tous les jeunes gens de ce territoire allemand de quelque cent mille kilomètres carrés situé entre l'Elbe et l'Oder, la confrontation au passé brun de son peuple avait été relativement approfondie et s'était effectuée avec le large soutien de l'État. [...] Mais 
cela lui suffisait-il ? N'était-ce pas là un simple matériau scolaire qu'il avait été bon gré, mal gré forcé d'assimiler? N'étaient-ce pas des faits qui venaient confirmer superficiellement une opinion déjà plus ou moins arrêtée mais n'incitaient pas à ressentir ou à réfléchir? Un travail resté à l'état d'ébauche donc?» (158). Si le jeune Schlesinger décerne ici un relatif satisfecit au travail de son pays sur le passé, il s'en démarque aussi clairement: au bout du compte, il lui reproche son caractère dogmatique et abstrait, et revendique qu'il soit mené à terme par l'investissement intellectuel et émotionnel de chacun dans la question : il doit s'agir d'un Nachfühlen et d'un Nachdenken, d'un retour sur le passé par le sentiment et la pensée.

31 Certes, Schlesinger prend aussi ses précautions et donne ici ou là des gages, mais toujours de façon à mieux avancer un pion derrière. S'il est dit que la "société » estallemande "s'est suffisamment protégée par la transformation des structures de propriété » (165), si bien que les Berger, « pour peu qu'ils ne soient pas passés de l'autre côté ", ne représentent plus de "menace potentielle», ce raisonnement théorique orthodoxe est immédiatement contrebalancé par le «devoir moral de mettre en marche une investigation » (165), donc de régler les comptes sur le plan individuel. De la même façon, Schlesinger se garde de faire du père un membre influent de la RDA ou un membre du SED. Le père de Michael est juste "directeur technique " d'une entreprise, un "sans parti ", qui, du point de vue des autorités communistes, "traîne encore avec soi quelques scories bourgeoises». Cependant, le texte le qualifie de "progressiste», donc d'allié du régime: $\mathrm{M}$. Berger père est un cadre apprécié du personnel, qui a obtenu «deux fois la distinction de meilleur ouvrier, deux fois celle d'activiste » (165).

Schlesinger imagine donc la carrière et la reconversion réussie en Allemagne de l'Est d'un ancien officier de la Wehrmacht impliqué dans des crimes nazis. Certes, il ne fait pas non plus du père un grand criminel de guerre. Ce qu'il thématise, c'est plutôt la culpabilité ordinaire d'un citoyen respecté de RDA : au bout du compte, Michael ne peut soupçonner son père que de la participation à une action de représailles, à laquelle il dit de surcroît " avoir juste assisté » (178). Mais s'il n'a pas la preuve que son père est un assassin, il est en revanche certain qu'il s'est rendu coupable de "complicité de meurtre » et qu'il n'a rien fait contre. À travers le cas Berger, le texte pose la question de la présence, jusqu'en RDA, de ces «dizaines de milliers » d'exécutants nécessaires à la mise en œuvre des décisions de Wannsee : « Combien [...] de Berger avait-il fallu pour assassiner [...] sept millions d'êtres innocents? Et où étaient-ils maintenant? S'appelaient-ils donc de nouveau [...] Monsieur Berger, habitaient-ils même dans l'immeuble d'à côté, étaient-ils assis face à nous dans le tramway, nous vendaient-ils le journal le matin? Vivaient, travaillaient-ils tout comme nous, dont l'alibi repose sur le hasard d'avoir été trop jeunes pour commettre de tels crimes? Vivaient-ils aussi avec les fils de leurs victimes... ?» (164).

Avec ses batteries de questions, ses «nous " générationnels, son pathos, le texte a des accents annonciateurs du conflit des générations et de la Väterliteratur des années 1970. Et c'est là que les audaces politiques, esthétiques et culturelles du texte se rejoignent : Michael, comme son jeune auteur, a commencé à remettre en cause "l'autorité de l'âge, de l'expérience » (179), le statut intangible de cette génération d'anciens résistants et de bons citoyens investis dans la construction du socialisme. Vers la fin du texte, dans une optique déjà soixante-huitarde (attentive au pouvoir de la langue et des mécanismes psychologiques), Michael s'aperçoit « pour la première fois de sa vie » que 
lui et son père "parlaient des langages différents" (179). Et il se cabre contre le " sentiment » d'être "soi-même en tort, soi-même accusé » (179): " comme si j'étais moi-même coupable, pour l'avoir confronté à ce qu'il croyait enterré, oublié » (177).

Au-delà de certaines concessions et circonvolutions tactiques, Schlesinger adresse donc un message clair à la RDA. Le monde radieux promis par le socialisme - symbolisé dès l'entrée du texte par le «bleu du ciel », " promesse de paix » et de bonheur - s'avère terni : il n'est plus un «vrai bleu », mais un « décor absurde » derrière lequel se cache «l'inconcevable» (156). De même, les « yeux magnifiquement bleus» de Michael sont devenus des "yeux désemparés », des "yeux torturés » (167). Un détail qui signale davantage qu'une simple perte d'illusions ou un simple déniaisement du héros: la découverte du passé a déréalisé les prétentions à l'innocence de la société antifasciste. Pour retrouver un vrai bleu, il faudrait que le travail de confrontation au passé «brun ", resté à l'« état d'ébauche » en RDA, soit mené à son terme. Certes, Michael n'a pas la solution: «Il ne voit que le compte non réglé, pas comment le solder» (181). Mais la foi dans un présent radieux n'est plus de mise, et la dernière phrase le souligne : au pied de l'immeuble de ses parents qu'il vient de quitter, il « se sent fatigué, éreinté. La journée est encore d'un bleu radieux » (181).

\section{Les enjeux du subjectivisme et du modernisme}

La réponse de H. Plavius est un exercice curieux, fascinant pour le lecteur d'aujourd'hui, où l'on voit ce rédacteur de la revue de l'Union des Écrivains avancer sur une corde raide, pris entre le désir de promouvoir une littérature moins monolithique et les limites imposées par la politique culturelle du régime. Soucieux de ne pas éveiller les soupçons d'autorités plus haut placées, il ne met d'ailleurs pas franchement en avant le sujet du récit, qu'il réduit à un "conflit " intérieur du héros, "situé " très euphémiquement « dans la sphère de l'humain et de sa négation» (182). C'est presque en passant qu'il mentionne "l'indignation de Michael sur les crimes du fascisme" (183). Il donne en outre très vite un gage majeur en soulignant que ce dernier choisit de ne pas passer à l'Ouest à une époque où l'ouverture des frontières le permettait encore. Et, à cette occasion, il fait un hors sujet magistral: "car tous ceux qui, aujourd'hui encore, en Allemagne, se dérobent à la maîtrise du passé seront forcés de s'exécuter demain » (183). Superbe façon de botter en touche - ou plutôt de l'autre côté du mur -, comme si Michael était bien un citoyen scrupuleux de la RDA, mais que son père criminel résidait, lui, « en Allemagne ».

Sa stratégie consiste donc à estomper les audaces de fond au bénéfice de certaines audaces formelles - qui d'ailleurs ne dépassent guère le cadre de ce qui est déjà admis. Mais, la forme et le fond étant inséparables, il est contraint de reconnaître que le texte de Schlesinger «se risque souvent tout près de la limite " (190), à savoir la remise en cause subjective d'une maturité antifasciste supposée objective de la RDA. Ainsi, le " monologue intérieur [...] n'évite pas ici une faiblesse, à savoir de fonctionner comme l'expression d'une certaine passivité et indécision [...] » (185). Le héros n'est pas assez positif ou ne subit pas une transformation assez claire: Plavius relève "l'absence de but de ses pensées et de ses actes ", le « désarroi » dans ses yeux, son "incapacité à parvenir à une décision claire" (185). Conjuguées au subjectivisme formel, les « limites» du héros (184) font que le texte flirte avec les «dangers de l'esthétisme ». Par sa « fin ouverte » (186), menace d'« indétermination » dans la signification ultime 
du personnage, le texte n'est pas loin de prêter le flanc à des accusations de «modernisme » (188), d'abstractionnisme ou d'attirance pour « l'absurde » (190). Bref, l'« intention de l'auteur » (184) n'est pas assez claire : « Michael n'apparaît pas encore comme un personnage qui exprime le degré de maîtrise sur le monde environnant atteint par la société dans son ensemble dans notre république » (186). Le reproche est classique : le héros n'est pas assez mûr politiquement, les tourments de sa subjectivité ne sont pas suffisamment contrebalancés par l'objectivité de solutions collectives déjà mises en œuvre par le socialisme.

Schlesinger ne s'y trompe pas. Rejetant dans sa réponse la notion de «passivité » de son héros, il écrit : « Michael est aussi actif qu'il peut l'être dans sa situation. La pensée, les processus de confrontation intérieure sont également des critères d'activité » (190-91). Il réaffirme ainsi ses prémisses : le travail sur le passé, pour être accompli, devra passer par un investissement de la subjectivité. Et c'est là que, de nouveau, forme et fond se rejoignent, car les formes de cette "confrontation intérieure " en littérature ne pouvaient à terme que se heurter aux dogmes de l'antifascisme et du réalisme socialiste. Le potentiel subversif du texte dans le contexte de la RDA ne résultait pas tant d'un certain esprit bohémien ou anticonformiste ${ }^{31}$ que de la démarche même de confrontation au passé, qui plaçait Schlesinger dans un processus d'émancipation littéraire, idéologique et politique. Pour reprendre un terme d'Emmerich désignant l'émergence d'aspirations largement partagées dans les milieux intellectuels de ces années-là, il était « en route vers l'objection »"

\section{Le contexte (1963-1965)}

38 C'est d'ailleurs bien le diagnostic que fait le pouvoir, qui, six mois plus tard, en décembre 1965, étouffe ces aspirations lors du fameux $11^{\mathrm{e}}$ plénum du Comité central du SED. Rétrospectivement, il apparaît que le texte de Schlesinger est publié juste à temps : juste avant que s'achève une période que lui-même et un certain nombre d'acteurs de la vie culturelle est-allemande avaient pu percevoir comme plus ouverte. Dans Fliegender Wechsel, Schlesinger revient sur l'adoption du NÖS en janvier 63, ce « nouveau système économique de planification et de direction » censé donner davantage d'autonomie aux acteurs économiques et qui, après un premier accueil sceptique, suscita d'après lui un « espoir secret après toutes ces années de déprime qui avaient suivi le soulèvement en Hongrie et l'isolement consécutif à la construction du Mur » (105). Dans un entretien de 1996, il parle d'un « climat plus ouvert ». «Lisez donc la presse des années 1963 à 1965 jusqu'au $11^{\mathrm{e}}$ plénum ! Je crois que ce fut sa meilleure époque en $\mathrm{RDA}^{33}$.» Et, en 2001 : «Le journalisme des années soixante-quatre, soixante-cinq, en RDA, avait un niveau élevé pour l'époque. Je suis retourné voir plus tard ce que nous faisions à l'époque : il n'y avait rien là d'absolument éternel, mais, pour l'époque, c'était un travail important, de qualité, et qui brisait même en partie certains tabous $\rrbracket^{34}$.

Et il est vrai que la période est marquée par la parution et l'éloge très officiel de livres relativement novateurs (Der geteilte Himmel de Christa Wolf, Ole Bienkopp de Erwin Strittmatter ou Die Spur der Steine de Erik Neutsch) ou par les débats consécutifs à la conférence Kafka de Liblice (mai 1963), qui se solde par la première édition de ses œuvres en RDA en 1965. Pour ne mentionner que la NDL, qui se verra publiquement tancée par Honecker lors de son discours au $11^{\mathrm{e}}$ plénum $^{35}$, elle publie en mars 1965 des extraits d'un colloque international d'écrivains qui s'est tenu en décembre 1964 à Berlin 
Est (dont un texte de son rédacteur en chef, Wolfgang Joho, admettant que les accusations de "décadence " procédaient parfois d'une méconnaissance de l'œuvre et des extraits d'un discours de Hermlin critiquant les atteintes dogmatiques à la « liberté artistique $)^{36}$. NDL prend même quelques risques supplémentaires en publiant un extrait de Rummelplatz, de Werner Bräunig, roman qui abordait les tabous de l'alcoolisme et de la sexualité en milieu ouvrier et qui fut mis à l'index en même temps que la revue par Honecker.

Le climat plus ouvert qu'évoque Schlesinger est cependant resté très circonscrit dans le temps et très relatif. La période 1963-65 est marquée par des mises au point répétées d'Ulbricht et des plus hautes autorités culturelles sur le rôle dirigeant du parti ${ }^{37}$ et par l'adoption simultanée de mesures de répression ${ }^{38}$, cette succession et coexistence même des phases constituant, avec son arbitraire, un mode de contrôle des milieux intellectuels : ainsi, Emmerich parle d'une forme d'« autoritarisme calculé » du régime à l'égard des écrivains dans la décennie suivant la construction du Mur ${ }^{39}$. Mais les niches de liberté surveillée pouvaient coexister avec leur contraire. Dans l'entretien de 2001, Schlesinger estime que la vie culturelle en RDA n'était pas monolithique, du fait par exemple de la « concurrence » entre les sections «Culture » et «Propagande » du Comité central et de l'existence de "différences» jusqu'au sein du parti unique entre des « conservateurs » et des «libéraux ». S'abstenant de juger des années 1980, où il vivait à l'Ouest, Schlesinger avance que la RDA, « dans les années 1960 et 1970 », n'était pas « un État homogène, contrôlé de bout en bout »" .

41 Ces deux années d'ouverture relative sont en tout cas fructueuses pour l'écrivain débutant. Inscrit depuis le printemps 1963 aux cours de reportage de Jean Villain ${ }^{41}$, détenteur en parallèle d'un contrat de travail auprès de la Neue Berliner Illustrierte, il participe au projet de création d'un magazine d'information socialiste inspiré du Spiegel, baptisé « Profil ». Ce projet bénéficie du soutien, au sein du Comité Central, du rédacteur en chef de la NBI, Hans Otten, et se voit encourager par Willi Stoph, alors président du Conseil des ministres, qui félicite Villain par télégramme pour le numéro pilote $^{42}$. Dans ce cadre, Schlesinger publie une dizaine de reportages, avec une prédilection pour le social ${ }^{43}$ et la question du passé : un long et ambitieux article sur le cimetière juif de Berlin-Weißensee ${ }^{44}$, un reportage antimilitariste sur le déminage d'un champ de tir dans une forêt de la Marche de Brandebourg ${ }^{45}$. La période, cependant, se referme brutalement pour lui aussi. Le cours de Villain est fermé dans les semaines menant au plénum, le projet Profil, déjà gelé en 1964, voit son numéro zéro classé «confidentiel $»^{46}$ et son ouvrage sur $«$ l'art du reportage » interdit et mis au pilon ${ }^{47}$. Schlesinger est renvoyé de la NBI et promis à une relégation "dans la production ", à un poste de rédacteur d'entreprise ${ }^{48}$.

Rétrospectivement, Schlesinger ne manque pas de sévérité dans le jugement qu'il porte sur lui-même et sa "proximité avec le système dans les années 1960 » $^{49}$. Il parle ainsi d'une «naïveté négligente» dans son appréhension des signes de raidissement du pouvoir ${ }^{50}$. Mais ces années de formation - cours, reportages, écriture - lui ont également appris, dit-il, à mieux voir les « dissonances réelles, [...] les contradictions » du système $\mathrm{e}^{51}$. Mieux : par l'écriture, il est entré dans une démarche de confrontation subjective au passé - et par là au présent socialiste. C'est bien la question centrale de ces années-là, parfaitement résumée par Christa Wolf lors du $11^{\mathrm{e}}$ plénum, où, défendant seule l'extrait de Bräunig publié par NDL, elle affirmait que «l'art ne peut pas renoncer à être subjectif $\aleph^{52}$. 
43 Et c'est bien la question qui accompagne Schlesinger dans la deuxième moitié des années 1960. Évoquant dans Fliegender Wechsel les répercussions du $11^{\mathrm{e}}$ plénum sur son existence, il raconte comment, après avoir dû quitter la NBI, il a été contacté au printemps 1966 par Kurt Batt et Konrad Reich, respectivement lecteur et directeur de la maison d'édition Hinstorff, lesquels lui ont proposé une bourse d'écriture pour finir la rédaction de "Michael ». Il retrace ses premières ébauches, neuf mois de brouillons et premiers jets infructueux - «trois douzaines de débuts [...], rien que du papier, un verbiage affecté, tout s'effilochait, rien ne vivait vraiment» - puis la pression croissante des échéances, en particulier un «bilan de mi-parcours » prévu à l'occasion d'une conférence des auteurs subventionnés. Et, enfin, à la table d'un café, l'inspiration : «quelque part entre la deuxième ou la troisième gorgée [...], je couchai une phrase sur le papier, et cela démarra, ce sentiment que je ne connaissais que de mon enfance [...], toutes les phrases, toutes les images, toutes les idées s'ordonnaient, je pris tout en notes, avalai d'une traite le reste de mon café, rentrai chez moi par le soixante-douze, parvins à mettre au point un début et dix pages vers le milieu pour la conférence, je fis ma lecture, je vis Batt rayonner, j'obtins sur le champ une prolongation de ma bourse, avec la sensation plutôt que la certitude d'avoir commencé là à être un écrivain - tout cela alors que, dans ce café, je n'avais guère fait que constater, fort banalement, que je me trouvais dans un café. Mais j'avais dit je. Je ${ }^{53}$. »

\section{Le roman, Michael (1971)} qu'un organisme officiel polonais finit par démentir formellement que l'officier sur la photo puisse être le père de Michael. Le lecteur, cependant, n'apprend l'existence de ce démenti qu'au tiers du roman, et lit donc d'abord le texte comme le récit d'un règlement de comptes avec un père criminel et comme un corps à corps émotionnel avec les conséquences de la rupture - puis, dans un deuxième temps, comme une confrontation à la culpabilité plus ordinaire d'un officier de l'armée allemande. Disculpé de ce crime précis, le père n'est pas blanchi : sa participation à la guerre, sa 
possible présence passive lors d'exactions de l'armée demeurent, et le texte les réinscrit dans le cadre plus large de la Shoah.

Michael, en effet, passe en revue l'éventail des comportements possibles de l'époque et explore les conséquences émotionnelles de ces configurations pour la génération suivante : l'implication dans des crimes contre l'humanité, la complicité plus ou moins passive, le suivisme enthousiaste ou désabusé. Il explore également des configurations non réellement advenues et pourtant centrales, comme la disparition du père, vécue un moment comme réelle à la fin de la guerre avant d'être démentie par son retour de captivité. Le cas du père est relié à des personnages parallèles : le cas d'un médecin SS actif dans les camps d'extermination auquel le narrateur, qui, lycéen, a assisté à une demi-journée de son procès avec sa classe, ne peut s'empêcher d'associer son père; le cas aussi d'un ami officier ayant payé de sa vie un acte de résistance de la dernière heure. Ce que le texte explore, c'est donc l'éventail des comportements d'un peuple dans les dernières années de la guerre. Et il est logique que le texte débouche sur la question de la complicité du narrateur, enfant de huit ans en 1945, mais fanatique du régime et du Führer. C'est à dessein que Schlesinger donne son année de naissance à son héros. Le roman a une dimension introspective marquée où l'auteur revient sans complaisance sur son propre passé d'«enfant de la race des seigneurs» (155) et sa propre vénération du régime, incarnés à la fois par la figure du père et celle d'un Führer adulé.

Michael est donc une invitation faite à chaque lecteur à se confronter non seulement à sa propre histoire, mais également à tout l'éventail de ces cas de figure. La complexité et la non-linéarité du texte sont évidemment le reflet du travail de mémoire, d'introspection et de reconstruction de soi rendu nécessaire par l'irruption des crimes dans la conscience de l'individu. Mais elles sont aussi la réalisation dans le récit même d'une phrase de l'esquisse de 1965 entre-temps disparue du roman: "La vérité est compliquée. Peut-être se situe-t-elle au centre de tous les possibles»(165). La confrontation au passé ne pourra réussir que si elle est à la fois complexe et subjective, si elle mêle examen familial, national et individuel des culpabilités. Et, à cet égard, on mesure la force de l'équation personnelle mise en place dans le récit: l'auteur, qui a perdu son père dans la dernière semaine de combats, le fait revivre dans une fiction pour se confronter à lui dans tout l'éventail des possibles laissé ouvert par sa disparition. Y compris en y ménageant une place au complexe de sentiments engendré par sa mort.

Dans le contexte de la RDA, la nouveauté de Michael consiste dans cet appel à mener à bien l'examen du passé au niveau des citoyens ordinaires. Il est symptomatique que le résistant à l'aune duquel le père est jugé ne soit pas un ancien déporté antifasciste mais un résistant de la dernière heure, un jeune officier dénommé Gessener, séduit dans son adolescence par le régime puis poussé dans les derniers jours de la guerre à un acte de désespoir pour sauver sa conscience. Il y a certes une figure de résistant véritable dans le roman, un collègue dénommé Kapinski auquel le narrateur se confie, ancien déporté politique qui conserve dans son vestiaire une tenue rayée, avale sans cesse des " cachets » et travaille à des " notes " pour un institut historique sur ses expériences à Sachsenhausen et «le travail du comité secret de la résistance » (44-46 et 186). Mais si Michael se confie à lui, il ne mesure son père qu'à Gessener. Kapinski, au fond, fait figure d'intouchable. 
50 À travers ce personnage, c'est toutefois un débat essentiel qui se joue. D'abord parce que Kapinski aborde la question de la culpabilité sous l'angle des «structures économiques » et que, hostile à toute "surévaluation des conflits individuels » (45), il considère les «décisions " concrètes des individus à la guerre comme « superfétatoires » (186), tendant ainsi, à l'instar du régime, à dédouaner les habitants de la RDA. Citant Brecht et le finale de l'Opéra de quat'sous, il prône l'indulgence pour les "petits méfaits individuels" par opposition aux "grands méfaits» (45), face à quoi Michael se dit «préoccupé par de simples questions de coexistence, par la question de savoir comment côtoyer à table un homme sur l'intégrité morale de qui j'ai de forts doutes » (45).

51 Ensuite, parce que Schlesinger le montre embourbé dans son propre rapport au passé. Les «bouts de papier » couverts d'une écriture «petite » et "serrée » qui parsèment son bureau symbolisent un travail douloureux mais trop abstrait pour être cathartique : tout à sa quête d'une vérité objective, il reste avec sa douleur individuelle, qu'il soigne à coups de cachets. Au détour d'une conversation, il «penche la tête sur ses bouts de papier [...], c'est sa vie, sa vie à lui, Kapinski, couchée sur des fiches à l'écriture serrée, il penche la tête donc, et dans ses yeux surgit une trace de fatigue, parfois je te comprends quand même " (45). L'accès de faiblesse laisse vite la place à l'« acuité perçante » de son regard, mais l'aveu d'impuissance est là. Kapinski représente la face humaine d'un antifascisme trop abstrait qui, occupé à disserter sur «la nature influençable de masses désorientées » ou « les erreurs tactiques du parti vers la fin des années vingt », ne soigne pas les cicatrices de l'individu (45).

52 L'examen du passé, du coup, débouche sur le présent. La passivité du héros critiquée par Plavius est présentée ici justement - y compris pour Kapinski et ses accès de découragement - comme le résultat d'un rapport non élucidé aux traumatismes du passé. Ainsi, pour Michael, l'incapacité à parler remonte à la fin de la guerre. Son silence - lors du départ du père pour le front dans les derniers jours de combat, lors de son retour inespéré quelques années plus tard, lors de la rupture avec lui en 1960 - est le même que celui qui pousse sa petite amie Thea à partir en Allemagne de l'Ouest et qui l'empêche à la fois d'agir et de trouver les mots pour la retenir. Aux demandes d'explication de Michael, elle répond: "Peut-être le calme [...], peut-être est-ce ton calme que je ne supporte pas » (197). Se souvenant du moment de son départ, à la gare, le narrateur se dit: "Je crois ne l'avoir jamais vue aussi désemparée, et peut-être qu'elle serait vraiment restée si j'avais su trouver les mots » (196).

53 À travers la passivité et la non-parole d'un citoyen de RDA, Schlesinger fait le diagnostic d'un travail de deuil éludé et de l'incapacité afférente à s'approprier son destin dans l'histoire. Inversement, au bout de l'exploration du passé, il y a pour Michael la découverte de la parole, l'exercice de la subjectivité et la question d'une construction autonome de l'existence. À Michael qui ne comprend toujours pas, Thea répond en disant qu'il s'agit de savoir « ce qu'on fait, ce qu'on veut, la façon dont on vit, à chaque instant » (197).

54 Dans un compte rendu du roman pour NDL, en 1972, Plavius voit bien que le texte débouche sur " le présent ", et il le range dans une " série d'œuvres en prose » récentes dont le sujet tourne autour de « la question de la connaissance et de la réalisation de soi $»^{55}$. Certes, il reproche encore au texte son côté trop « existentiel », un « déficit » de solutions viables, collectives ou "sociales » plutôt qu'« individuelles», qui seraient ancrées dans un rapport « constructif » au monde du «travail », mais il comprend bien 
que le roman est porteur d'aspirations plus générationnelles à l'émancipation. Au fil d'une démarche annonciatrice des grandes introspections sur le passé de la RDA (p. ex. Kindheitsmuster de Christa Wolf) et de la Väterliteratur occidentale, Schlesinger s'est peu à peu affranchi de l'« autocensure ", " cette instance de contrôle contre laquelle ", dit-il, « je me suis si désespérément battu en écrivant mon premier livre $»^{56}$. Dans un entretien non publié de 1975, quatre ans après la sortie de Michael, il confiait à Frank Hörnigk : «Écrire est devenu pour moi une espèce d'acte d'émancipation ${ }^{57}$. Pour la singularité de ce processus d'émancipation par l'écriture et l'intégrité de sa démarche de confrontation au passé, il est urgent de redécouvrir cet auteur ${ }^{58}$.

\section{NOTES}

1. Cahier 11, 1960. Réédité dans Klaus Schlesinger: Die Seele der Männer, Aufbau Taschenbuch Verlag, 2004, pp. 99-112.

2. « Michael. Entwurf einer Erzählung », Neue Deutsche Literatur, cahier 150, mai 1965.

3. Rostock, Hinstorff Verlag, 1971. Paru également à l'Ouest en 1972, sous le titre Capellos Trommel, Zurich, Cologne, Benziger Verlag.

4. «'Deshalb ist Literatur immer eine Form der Freiheit...'. Ein Gespräch mit Klaus Schlesinger », in die horen, $\mathrm{n}^{\circ} 201$, Bremerhaven, 2001, réédité dans Die Seele..., op. cit., p. 333.

5. «Das doppelte Ich», in: Klaus Schlesinger: Von der Schwierigkeit, Westler zu werden, Aufbau Taschenbuch Verlag, 1998, p. 88.

6. « Mir meine Neurose von der Seele geschrieben », « Deshalb... », art. cit., p. 358.

7. Wolfgang Emmerich: Kleine Literaturgeschichte der DDR. Erweiterte Neuausgabe, Aufbau Taschenbuch Verlag, 2005, pp. 319-20.

8. Paul O'Doherty: The Portrayal of Jews in GDR Prose Fiction, Rodopi, Atlanta, 1997.

9. Claudia Mauelshagen: Der Schatten des Vaters: deutschsprachige Väterliteratur der siebziger und achtziger Jahre (Marburger germanistische Studien vol. 16), Francfort/Main (etc.), Lang, 1995, pp. 67-69.

10. Astrid Köhler: Brückenschläge. DDR-Autoren vor und nach der Wiedervereinigung, Göttingen, Vandenhoeck \& Ruprecht, 2007, pp. 49-74.

11. «Deshalb... », art. cit., p. 333.

12. Les numéros de page mentionnés dans le corps de l'article renvoient aux éditions citées.

13. Tatiana Berenstein et al (dir.), Rütten \& Loening. Une édition paraît la même année en RFA (Rödergerg Verlag, Francfort/Main), mais c'est l'ouvrage est-allemand que Schlesinger a eu en sa possession.

14. Faschismus, op. cit., p. 473.

15. Cf. sur le sujet les articles de Thomas Jung: « Nicht-Darstellung und Selbst-Darstellung: Der Umgang mit der 'Judenfrage' in der SBZ und der frühen DDR und dessen Niederschlag in Literatur und Film », in Monatshefte Wisconsin, 90-1, 1998 et « Aus den Schatten der Vergangenheit treten: Das Schreiben jüdischer Autoren aus der DDR vor und nach der Wende ", in Roswitha Skare et Rainer B.Hoppe (dir.), Wendezeichen?: Neue Sichtweisen auf die Literatur der DDR, Amsterdamer Beiträge zur neueren Germanistik, Rodopi, 1999. Après la dissolution forcée de la VVN en 1953 dans le sillage des procès de Prague et de Moscou et son remplacement - si révélateur - par le Comité des combattants antifascistes de la résistance (Komitee der 
Antifaschistischen Widerstandskämpfer), les publications sur la Shoah connaissent une période creuse qui contribue à « repousser durablement la perspective des victimes juives à l'arrièreplan » (Simone Barck: «'Grundfrage: Antifaschistischer Widerstand'. Zur Widerstandsrezeption in der DDR bis 1970 ", in UTOPIE kreativ, cahier 118 (août 2000), p. 789). Après ces années de "refoulement» ou de "tabouisation de l'histoire des souffrances et de la résistance juives » (Jung, "Schatten », p. 71), la sortie de Faschismus, op. cit., coïncide avec une certaine reprise des publications autour de 1958, date anniversaire du pogrom de 1938, marquée entre autres par la sortie du Journal d'Anne Franck, du roman de Bruno Apitz Nackt unter Wölfen ou de Im Feuer vergangen. Tagebücher aus dem Ghetto, mit einem Vorwort von Arnold Zweig, Ruetten \& Loening, 1958. 16. C'est une idée de fond. En 1953, lors de l'auto-dissolution de la VVN imposée par le Comité central du SED, le secrétaire général de l'association écrit: le «développement d'un ordre antifasciste et démocratique en RDA a [...] conduit à l'extermination de toutes les racines du fascisme, et la VVN a ainsi accompli sa mission historique». Cité d'après Jung: "NichtDarstellung ", art. cit., p. 56 (réf. : Verbandschreiben des VVN, février 1953, Dokumente SAMPOBA, DY 55/V 278 1/2).

17. Notons la redoutable polysémie des termes grâce auxquels s'opère le glissement: « nebenher " peut être traduit par «à côté », « en plus », « en passant », ou « accessoirement ». Et «Opfer » peut signifier sacrifices, mais aussi victimes.

18. Ces continuités sont également dénoncées en RFA par certains intellectuels et écrivains, mais la critique n'y va pas de soi. Schlesinger, pour sa part, dit avoir vu certains de ses commentaires sur la question censurés par le Beuth-Spiegel, « Gespräch... », art. cit., p. 337.

19. Klaus Schlesinger: «Verbrennungskommando Warschau », humanitas, $2^{\mathrm{e}}$ année, 26.07.1962, compte rendu du livre du même nom de Tadeusz Klimaszewski, Berlin, Verlag Volk und Welt, 1962

20. Bernard Mark: Der Aufstand im Warschauer Ghetto. Entstehung und Verlauf, Berlin, Dietz Verlag, 1957, en part. pp. 353-56.

21. Mitteldeutscher Verlag, 1958. Sommet de la littérature antifasciste, ce roman est en même temps un exemple de la marginalisation des Juifs dans la peinture littéraire des camps et de la hiérarchisation des résistances. L'enfant y est décrit de façon récurrente comme un petit animal, « insecte » $(19,22)$, « ver blanc » $(24)$, « chiot » $(54)$, ou comme une « chose » ou une "créature étrange » (22), et les déportés juifs comme un «amas » ou une «masse » d'individus passifs et pitoyables, «hommes sans nom et sans visage» "dont nul ne devinait les pensées et qui parlaient une langue que personne ne comprenait» (10), citations d'après l'édition Aufbau Taschenbuch (9e éd., 2006).

22. Cf. les articles de Jung, l'ouvrage de D'Oherty et Stephan Braese et al. (dir.): Deutsche Nachkriegsliteratur und der Holocaust, Frankfurt/New York, Campus, 1998.

23. En 1960, Schlesinger ne connaît pas le rapport de Hannah Arendt sur le procès Eichmann (1961) ni son concept de «banalité du mal » (Eichmann in Jerusalem: A Report on the Banality of Evil, New York, Viking Press, 1963).

24. À la fin de « Michael », on trouve cette mention : « écrit en 1960 ». Dans Fliegender Wechsel, op. cit., p. 108, Schlesinger indique plutôt avoir fini le texte en 1963.

25. Cf. Christoph Weiß: «'... eine gesamtdeutsche Angelegenheit im äußersten Sinne...' Zur Diskussion um Peter Weiss' Ermittlung im Jahre 1965 », in : Nachkriegsliteratur, op. cit., pp. 53-70.

26. Braunbuch. Kriegs- und Naziverbrecher in der Bundesrepublik, Berlin, Staatsverlag der DDR, 1965.

27. Le sujet, pour Schlesinger, représentait à l'époque une "violation de tabou », «Deshalb... ", art. cit., p. 335.

28. Schlesinger utilise deux fois le terme « absichernd » pour qualifier le texte de Plavius, laissant entendre qu'il s'agissait bien de «couvrir » la publication, Fliegender Wechsel, op. cit., p. 231, et « Deshalb... », art. cit., p. 335. 
29. Günter Erbe: Die verfemte Moderne: die Auseinandersetzung mit dem "Modernismus» in Kulturpolitik, Literaturwissenschaft und Literatur der DDR, Opladen, Westdeutscher Verlag, 1993, p. 80.

30. Schlesinger écrit « wieder zu vereinigen ».

31. Même si Honecker, dans son fameux discours lors du $11^{\mathrm{e}}$ plénum, condamne la « dégradation morale » d'une culture jeune «influencée » par l'Occident, $c f$. Elimar Schubbe (dir.): Dokumente zur Kunst-, Literatur- und Kulturpolitik der SED, Stuttgart, Seewald Verlag, 1972, pp. 1076-81.

32. Kleine Literaturgeschichte..., op. cit., p. 174.

33. « Kein magisches Datum », entretien avec Klaus Schlesinger, Freitag, ${ }^{\circ} 47$, du 15.11.1996, p. 9.

34. « Deshalb... », art. cit., p. 334.

35. Dokumente, op. cit., p. 1079.

36. Cf. sur le sujet Erbe, op. cit., pp. 80-85.

37. La lecture de Dokumente, op. cit., ne serait-ce que pour l'année 1964, est à ce sujet éloquente.

38. $C f$. le sort réservé à Havemann, Biermann, aux textes de Kunert ou au projet «Profil » (cf. infra).

39. Emmerich: Kleine..., op. cit., p. 179.

40. « Deshalb... », art. cit., p. 336.

41. Il s'agirait, et c'est tout un symbole, du seul cours sur le reportage dispensé dans l'histoire de la RDA. Cf. l'entretien de J. Villain avec Freitag, 15.03.2002.

42. Ibid. Le projet fut gelé juste après, suite à la destitution de Khrouchtchev en oct. 64 .

43. Par exemple des reportages sur le dernier centre pour sans-abri de Berlin ou sur le mont-depiété (Cf. « Deshalb... », p. 334).

44. « Fragen, gestellt aus einem Grab. Reportage », Neue Berliner Illustrierte 13, 1965, pp. 20-24

45. « Der verbotene Wald», Neues Deutschland du 3 avril 1966.

46. «Deshalb... », p. 335, Fliegender Wechsel, p. 110.

47. Die Kunst der Reportage, Verband der deutschen Journalisten, 1965.

48. Cf. Freitag et Fliegender Wechsel, p. 115.

49. Ibid., p. 104.

50. Ibid., p. 110.

51. Ibid., p. 109.

52. Contribution de Christa Wolf au $11^{\mathrm{e}}$ plénum, publiée dans Neues Deutschland, $\mathrm{n}^{\circ} 348$, 19.12.1965, repris dans Dokumente, op. cit., p. 1099.

53. Fliegender Wechsel, p. 233.

54. Pour une analyse plus détaillée du roman, $c f$. Daniel Argelès: « Mémoire et littérature : la confrontation au père et aux crimes du III ${ }^{e}$ Reich chez l'écrivain Klaus Schlesinger (1937-2001), in Carola Hähnel-Mesnard, Marie Liénard-Yétérian, Cristina Marinas: Culture et Mémoire. Représentations contemporaines de la mémoire dans les espaces mémoriels, les arts du visuel, la littérature et le théâtre, Palaiseau, Éditions de l'École Polytechnique, 2008.

55. Heinz Plavius: « Vom Entwurf zur Ausführung. Klaus Schlesinger: 'Michael', Hinstorff Verlag, Rostock », NDL, 1972, cahier 1, pp. 156-160.

56. Fliegender Wechsel, p. 104.

57. Frank Hörnigk: «Interview mit Klaus Schlesinger », 9. 12. 1975, archives Klaus Schlesinger, Akademie der Künste, Berlin, b. 91., p. 3.

58. Nous remercions pour leur aide l'Akademie der Künste de Berlin, ainsi que Michel Multan au prêt entre bibliothèques à l'École Polytechnique. 


\section{RÉSUMÉS}

Avec ses trois premiers textes, la nouvelle «David» (1960), l'ébauche de nouvelle « Michael » (1965) et le roman du même nom (Michael, 1971), Klaus Schlesinger (1937-2001) est à ranger parmi les premiers écrivains est-allemands à s'être confronté non seulement au passé nationalsocialiste, mais également à la politique nazie d'extermination des Juifs, et dans ce cadre à la question de la culpabilité individuelle des "pères » et de son héritage par la «deuxième » génération. Nous montrerons - par une analyse textuelle et par un examen des débats implicites ou explicites entourant ces textes - à la fois combien l'approche de Schlesinger était ancrée dans le contexte de la RDA, en particulier dans une certaine tradition " antifasciste », et combien elle s'en est émancipée sur les plans personnel, esthétique et politique, combinant modernité narrative et forte implication subjective pour parvenir à une confrontation exigeante et réussie au passé.

Mit seinen ersten drei Texten - der Erzählung «David» (1960), dem Novellen-Entwurf « Michael » und dem gleichnamigen Roman (Michael, 1971) - zählt Klaus Schlesinger (1937-2001) zu den ersten ostdeutschen Schriftstellern, die sich nicht nur mit der nationalsozialistischen Vergangenheit, sondern auch explizit mit dem Völkermord auseinandergesetzt haben und die sich in diesem Rahmen die Frage der individuellen Schuld der Väter und der Erbschaft dieser Schuld durch die «zweite » Generation gestellt haben. Anhand einer Analyse dieser Texte und einer Untersuchung ihrer Beziehungen zum ideologischen Umfeld versucht dieser Artikel zu zeigen, inwiefern Schlesingers Arbeit im Kontext der DDR wurzelte - besonders in der antifaschistischen Tradition der Zeit - und inwiefern sie sich zugleich persönlich, ästhetisch und politisch davon emanzipiert hat, denn diese anspruchsvolle Auseinandersetzung mit der Vergangenheit konnte erst anhand moderner und subjektiver Erzählstrategien erreicht werden.

\section{INDEX}

Mots-clés : passé national-socialiste, RDA

\section{AUTEURS}

\section{DANIEL ARGELÈS}

École Polytechnique (Paris) 